This is a postprint version of the following published document:

Parker, K., Schneider, R. T., Siegel, R. W.,

Ozisik, R., Cabanelas, J. C., Serrano, B.,

Antonelli, C. \& Baselga, J. (2010). Molecular

probe technique for determining local thermal

transitions: The glass transition at Silica/PMMA

nanocomposite interfaces. Polymer, 51 (21), pp.

4891-4898.

DOI: 10.1016/j.polymer.2010.08.051

(C) Elsevier, 2010

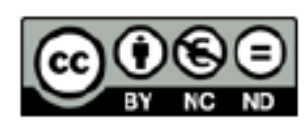

This work is licensed under a Creative Commons Attribution-NonCommercialNoDerivatives 4.0 International License. 


\title{
Molecular probe technique for determining local thermal transitions: The glass transition at Silica/ PMMA nanocomposite interfaces
}

\author{
Katelyn Parker ${ }^{a}$, Ryan T. Schneider ${ }^{a}$, Richard W. Siegel ${ }^{a}$, Rahmi Ozisik ${ }^{a}, *$, Juan Carlos Cabanelas ${ }^{\mathrm{b}}$, \\ Berna Serrano $^{\mathrm{b}}$, Claire Antonelli ${ }^{\mathrm{b}}$, Juan Baselga ${ }^{\mathrm{b}}$ \\ ${ }^{a}$ Department of Materials Science and Engineering, and Rensselaer Nanotechnology Center, Rensselaer Polytechnic Institute, Troy, NY 12180, United States \\ ${ }^{\mathrm{b}}$ Department of Materials Science and Engineering and Chemical Engineering, Universidad Carlos III de Madrid, Av. Universidad 30, 28911 Leganés, Madrid, Spain
}

\begin{abstract}
Local glass transition temperatures $\left(\mathrm{T}_{\mathrm{g}}\right)$ have been measured in the interfaces of solution blended silica/poly(methyl methacrylate) (PMMA) nanocomposites using florescence spectroscopy and compared with $\mathrm{T}_{\mathrm{g}}$ measured by differential scanning calorimetry (DSC). It was found that the two types of measurements yielded significantly different information. Combinations of silanes and poly(propylene glycol)-based molecular spacers bound to fluorophores were covalently linked to the surface of the nanoparticles, allowing for variation of the fluorophore response with respect to the distance from the nanofiller surface. Increases in the bulk $\mathrm{Tg}$ from the neat PMMA value were found upon the addition of nanofillers, but were independent of the nanofiller concentration when the filler concentration was above $2 \%$ by weight. Furthermore, as the size of the grafted molecular spacer was increased, $T_{g}$ values were found to decrease and approach $\mathrm{T}_{\mathrm{g}}$ of the neat PMMA.

Owing to variable conformations of the spacers, an effective distribution of fluorophore-silica distances exists, which influences the fluorophores' response to the transition.
\end{abstract}

\author{
Keywords \\ Glass transition \\ Nanocomposite \\ Fluorescence \\ spectroscopy
}

\section{Introduction}

The glass transition temperature $\left(\mathrm{T}_{\mathrm{g}}\right)$ is one of the characteristic properties that determines the functionality of a glassy material's use at a particular temperature, but a unified embodiment of the fundamental physics behind this transition has yet to be produced. Adam and Gibbs have introduced the idea of cooperative motion in which there is an intrinsic length scale over which cooperative dynamics takes place [1]. These domains rearrange into different conformations independent of the neighboring regions and are typically a few nanometers in size, but the length scale increases as the temperature is cooled to approach the $\mathrm{T}_{\mathrm{g}}$. Additional understanding of the Adam-Gibbs intrinsic length scale has been attempted through the investigation of confinement effects on polymer systems.

Initial studies examining confinement effects were performed by Jackson and McKenna on nanoporous glass filled with low molecular weight liquid [2]. Their results demonstrated that decreases in the $T_{g}$ were observed as the pore size decreased. However, the most important aspect of the glass transition in nanopores is the fact that the cooperatively rearranging region's relationship to the confining pore size. The cooperative behavior is described by a characteristic length scale that increases with decreasing temperature. However, when confined inside a nanopore, the characteristic length scale cannot be greater than the pore size; therefore, if the pore size is less than the characteristic length scale, the $T_{g}$ should always decrease because of the greater molecular dynamics experienced by the confined system compared to the unconfined (bulk) state. [3-5] This effect is termed as the "confinement effect". However, the interaction of the glass former with the confining substrate can lead to a change in the direction of $\mathrm{T}_{\mathrm{g}}$, which is termed the "adsorption" or "interface" effect. The ability to distinguish the effect of confinement from that of inter-face in systems confined in nanopores provides a very important tool to understand the physics behind glass transition. For a detailed discussion, the reader is directed to an excellent review article written by Alcoutlabi and McKenna in 2005 that discusses $\mathrm{T}_{\mathrm{g}}$ change in various geometries [6].

Keddie et al. initiated the study of polymer films by observing the $T_{g}$ of polystyrene (PS) films of varying thickness supported by silica substrates [7]. As the thickness of the film was decreased, the $T_{g}$ value also decreased from that of the bulk. Successive studies involved the study of thin and ultrathin supported and unsupported polymer films. Decreases[8-17] and increases [9,16-20] in the $T_{g}$ of the thin films were found based upon the interaction of the

\footnotetext{
* Corresponding author.

E-mail address: ozisik@rpi.edu (R. Ozisik).
} 
polymer with the substrate. $T_{g}$ increases found in certain systems, such as poly(2-vinyl pyridine) (P2VP) or poly(methyl methacrylate) (PMMA) supported on silica, were hypothesized to be a result of attractive interactions between the polymer and the substrate [21]. These increases were suggested to be the result of the existence of hydrogen bonds between the silanol group from the silica and the ester side group in PMMA or the nitrogen atom in P2VP. In addition to film thickness, factors such as molecular weight or processing effects have also been examined in thin and ultrathin films. For example, Mattsson et al. [8] have used free-standing ultrathin PS films with varying molecular weight to observe that, as the molecular weight of the same material increases, the behavior of the $T_{g}$ shifts to a linear trend at high molecular weights as opposed to the non-linear trend observed by Keddie et al. [7] for low molecular weight films.

While there have been few theories to describe the chain dynamics within confined polymer systems, several models have been generated based on the enhanced chain mobility at a freely standing interface or an interface with non-attractive interactions. This enhanced mobility at the surface causes a reduction in $T_{g}$ that impacts the surrounding material until, at a certain depth from the free surface; the $T_{g}$ of the bulk is recovered. To explain the $T_{g}$ deviations from the bulk, models developed to reflect this enhanced mobility have involved the use of mobile surface layers, [7,8] percolation of domains with slow dynamics [22], entropy fluctuations and capillary waves [20,23], intermolecular coupling of motion and the degree of entanglement [24,25], and surface mobility of chains based on the coil radius [26]. While these models are predictive for certain cases, a unified model predictive of all experimentally observed behaviors has yet to be developed.

Polymer nanocomposites have also shown deviations from the neat polymer $T_{g}$ values because of the interactions between the matrix and the nanoparticles as well as the confinement effects created by the nanoparticles. Krishnamoorti et al. [27] found complete suppression of cooperative dynamics associated with glass transition in polystyrene and polyethylene oxide intercalated nanocomposites with fluorohectorite. Ash et al. [28] have shown that interactions between alumina nanoparticles and PMMA led to a depression in $T_{g}$ that was dependent on the size and concentration of the nanofiller used. Based on Long and Lequeux's percolation theory,[22] Ash et al. proposed that a highly mobile interaction zone existed at the nanoparticle/polymer interface and acted to interrupt the percolation of slow domains as the temperature was decreased [28]. Chen et al. [29] using silica/poly(vinyl alcohol) composites showed that the interaction zone thickness depended inversely on the silica concentration, and ranged between $10.1 \mathrm{~nm}$ for $1.5 \%$ silica content and $6.3 \mathrm{~nm}$ for $9 \%$ silica content. Srivastava and Basu [30] studied gold/poly(methyl methacrylate), PMMA, nanocomposites where the gold nanoparticles were also capped with PMMA at varying concentrations. Interface thicknesses were calculated from SAXS experiments and were compared to a correlation length (indicative of cooperatively rearranging regions). A critical crossover interface thickness of $2.6 \mathrm{~nm}$ (which is similar to the correlation length) was identified below which $T_{g}$ was lower and above which $\mathrm{T}_{\mathrm{g}}$ was higher than the bulk $\mathrm{T}_{\mathrm{g}}$. Bansal et al. also determined the $T_{g}$ as a function of nanofiller content, and explained their findings through correlation of thin film thickness to the harmonic average of the interparticle spacing [31]. In related work, Rittigstein et al. have performed studies to correlate the effect of interparticle spacing with film thickness to determine how deeply into the matrix the interactions can be sensed. [19] By sandwiching polymers between two substrates and annealing the material, the $\mathrm{T}_{\mathrm{g}}$ was found to exhibit deviations for films hundreds of nanometers thick, indicating that even at low filler concentrations, confinement effects can be felt. To account for the $T_{g}$ changes in nanocomposite systems, Lee et al. developed a thermodynamic model incorporating both the Flory-Huggins theory and the configurational entropy model [32]. Deviations in the $T_{g}$ were found to be dependent on the intensity of the specific interaction entropy between the components of the nanocomposite, but while this model could be used to accurately predict $T_{g}$ values for systems involving decreases, the model could not be used to predict values for nanocomposites exhibiting constant $T_{g}$ over a range of filler concentrations.

Fluorescence spectroscopy has recently been developed to determine thermal transitions in polymer systems. Conventional techniques such as differential scanning calorimetry or ellipsometry record the response of a material while fluorescence spectroscopy records the response of specific fluorescent sites within a material. Because fluorescence is measured from specific molecules (fluorophores), the response of the system is based on the changes in the local environment, including physico-chemical changes in the rigidity and polarity of the system.

Torkelson et al. have taken advantage of this capability by monitoring the $T_{g}$ in patterned $[9]$ and multilayered $[16,17,33]$ films. In the multilayered films, thin layers of polymer chains labeled or doped with aromatic fluorophores were spin-coated on top of or between layers of polymer films to determine the transition in systems with different polymer-polymer interactions or at different depths within a certain polymer. In these studies, plots of the emission spectra maximum intensity versus temperature as well as the ratio of intensities at two different points on the spectra and the integrated intensities versus temperature were produced in which the glassy- and rubbery-state regions were linearly fitted at temperatures well before and after the $T_{g}$, respectively. The intersection of these fits was characterized to be the $T_{g}$ for the system. Another photophysical parameter used to characterize the $T_{g}$ is the first moment of emission band, $<\nu>$ :

$\langle\nu\rangle=\frac{\sum I_{\mathrm{F}}\left(\nu_{\mathrm{F}}\right) \cdot \nu_{\mathrm{F}}}{\sum I_{\mathrm{F}}}$

in which $I_{\mathrm{F}}\left(\nu_{\mathrm{F}}\right)$ is the fluorescent intensity at a given wavelength. $\langle\nu\rangle$ has been used both to observe in situ polymerization of epoxies [34-37], and the thermal transitions in epoxy/silica composites $[38,39]$. This parameter offers an easy method and gives a direct measure of the excited state average relaxation of the fluorophores. As a consequence, at often times it offers more sensitive detection of changes in polarity and rigidity than the maximum emission intensity.

Thermal transitions in both the bulk and local environments of PMMA/silica nanocomposites were measured in the present study. Extrinsic fluorescent probes were attached to hydrophobic chains of varying molecular weights to receive local information as a function of distance from the nanoparticle surface while classical DSC measurements were made to provide bulk $T_{g}$ values. Analysis of the thermal transitions from the fluorescence emission spectra was accomplished by examining the behavior of two photophysical parameters: the integrated intensity and first moment of the emission band, $\langle\nu\rangle$. In addition to varying the distance from the nanoparticle surface, the effect of the nanoparticle concentration was also investigated.

\section{Experimental}

\subsection{Materials}

Nanoparticles of silica $\left(\mathrm{SiO}_{2}\right)$ dispersed at $20 \mathrm{wt} \%$ silica in n,n-dimethyl acetamide (DMAc, under the commercial name DMAC-ST) were kindly donated by Nissan Chemical Industries, LTD. 
All silica concentrations reported throughout the current study are by weight. These nanoparticles had a reported diameter of $20.6 \mathrm{~nm}$ and specific surface area of $190 \mathrm{~m}^{2} / \mathrm{g}$. Particle size analysis was performed with the dynamic light scattering technique (DLS; Malvern ZetaSizer NanoZS Zen 3600) on 5\% silica samples that yielded a most probable particle size of $25.0 \mathrm{~nm}$ and an average size of $7.86 \mathrm{~nm}$.

Poly(methyl methacrylate) (PMMA, $M_{\mathrm{w}}=1.2 \times 10^{5} \mathrm{~g} / \mathrm{mol} ; 6 \%$ isotactic, 39\% atactic and 55\% syndiotactic; Aldrich), (3-aminopropyl)-dimethyl-ethoxysilane (APMES, 97\%; ABCR GmbH \& Co.), (3-glycidoxypropyl)-dimethyl-ethoxysilane (GPMES, 97\%; ABCR $\mathrm{GmbH} \&$ Co.), polypropylene glycol (Jeffamine D-230, $M_{\mathrm{w}}=230 \mathrm{~g} /$ mol; and Jeffamine D-400, $M_{\mathrm{w}}=400 \mathrm{~g} / \mathrm{mol}$; Fluka), dansyl chloride (DNS-Cl; Invitrogen), dansyl cadaverine (DNS; Biochemika) were used as received (see Fig. 1). Solvent grade DMAc ( $\geq 99.5 \%$; Sigma-Aldrich), methanol (Panreac), and tetrahydrofuran (THF) (multisolvent GPC grade; Scharlau) were used without further purification.

\subsection{Sample preparation}

\subsubsection{Surface modification of nanoparticles with silanes}

Silane coupling agents were added to the silica dispersion diluted with DMAc to give $5 \%$ silica solutions. The quantity of silane used was based on the reported specific surface area of the nanoparticles, the number of surface hydroxyls present on the silica nanospheres (4.6 hydroxils $/ \mathrm{nm}^{2}$ ) [40], and the molecular weight of the silane used. [28] To obtain a monolayer coverage of silane over the silica surface $0.234 \mathrm{~g}$ of APMES and $0.319 \mathrm{~g}$ of GPMES per gram of $\mathrm{SiO}_{2}$ needs to be used. Treated nanoparticles were dried under vacuum at $80{ }^{\circ} \mathrm{C}$ until all solvent was removed. The presence of silane on the nanoparticle surface was confirmed with the use of Fourier Transform Infrared Spectroscopy (FTIR; Perkin Elmer Spectrum GXFTIR System), while the silane coupling agent surface coverage was determined through Thermogravimetric Analysis (TGA; Perkin Elmer Pyris 1).

\subsubsection{Preparation of PMMA/Silica nanocomposites}

Standard samples without surface functionalization, labeled $N x$ for nanocomposite ( $x=2$ or $5 \%$ showing silica content), were synthesized to observe the effect of introducing a fluorescent probe into the nanocomposite. To prepare standard samples, silica dispersions of 1.0 and $2.5 \mathrm{~mL}$ were diluted with 3.0 and $7.5 \mathrm{~mL}$ of DMAc, respectively, and were added to 9.8 and $9.5 \mathrm{~g}$ of PMMA, respectively, to create samples of 2 and 5\% silica in the nanocomposite. The silica nanoparticles and the PMMA were mixed using a solvent (DMAc) to obtain good dispersion and distribution of nanoparticles in the polymer matrix.

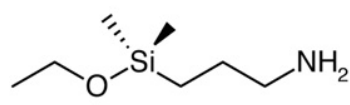

a

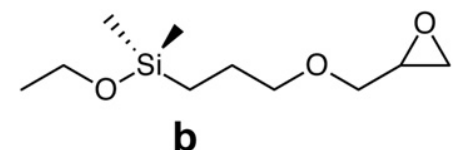

b

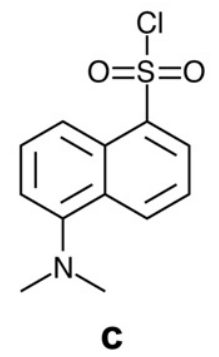

Fig. 1. Chemical structures of (a) (3-aminopropyl)-dimethyl-ethoxysilane, APMES, (b) (3-glycidoxypropyl)-dimethyl-ethoxysilane, GPMES, and (c) dansyl chloride, DNS-Cl.
The amount of APMES required to obtain monolayer coverage on the silica nanoparticles was reacted with DNS-Cl at a molar ratio of 40:1 in $1 \mathrm{~mL}$ of DMAc under constant stirring for $1 \mathrm{~h}$ to produce samples that were labeled $N x A D N S-C l$. Diluted silica dispersions (described above) were then reacted with the labeled silane molecules for $40 \mathrm{~min}$, at which point dynamic light scattering (DLS) measurements were taken to observe the stabilization of the reaction. PMMA was dissolved in the nanoparticle/DMAc mixture to stabilize the nanoparticle suspension and to obtain the proper silica/PMMA concentration.

For nanoparticles with long surface grafts, the appropriate amount of GPMES was added to both 2 and 5\% silica in DMAc and reacted under constant stirring for $1 \mathrm{~h}$. In a separate container, the appropriate amount of either Jeffamine D-230 or D-400 (5:1 M ratio of GPMES to Jeffamine) was added in a $4: 1 \mathrm{M}$ ratio to DNS-Cl and reacted under constant stirring for $1 \mathrm{~h}$. These two systems were then combined and reacted for $40 \mathrm{~min}$, at which point DLS measurements were taken. PMMA was then dissolved in the reaction mixtures to prepare 2 and 5\% silica nanocomposites, which were labeled as N2GJ230DNS-Cl and N5GJ400DNS-Cl, respectively.

\subsubsection{Nanocomposite processing}

Nanocomposite solutions were poured into a methanol bath and were allowed to equilibrate at room temperature for $2 \mathrm{~h}$. The resulting precipitates were then broken into small pieces, filtered with a Büchner funnel, and dried under vacuum at $80{ }^{\circ} \mathrm{C}$; this process was repeated once more to remove any residual DMAc. These dried samples were then compression molded at $190{ }^{\circ} \mathrm{C}$ for 30 min under $10 \mathrm{~kg}$ to form uniform samples. FTIR experiments on the used methanol was performed to determine the components in the bath fluid while TGA was used to quantitatively determine the composition of the remaining nanocomposite.

In order to monitor the fluorescent response of the PMMA matrix alone, two reference samples were prepared, one for DNS attached to APMES ( $R_{\text {Short }}$ ) and the other for DNS attached to D-400 Jeffamine chains $\left(R_{\text {Long }}\right)$. Adequate amounts of APMES and DNS-Cl to give a $1: 1 \mathrm{M}$ ratio of probe to spacer were dissolved in THF. PMMA was then added to this mixture, dissolved under constant stirring and vacuum dried at $80^{\circ} \mathrm{C}$ until all remaining solvent was removed, yielding a final probe concentration of $10^{-2} \mathrm{~mol} / \mathrm{kg}$. Preparation of the $R_{\text {Long }}$ was the same except for the solvent used that was chloroform instead of THF. These samples were also compression molded at the same conditions as the samples containing silica nanoparticles.

\subsubsection{Thermal analysis}

Differential scanning calorimetry (DSC) was used to determine the glass transition temperature $\left(\mathrm{T}_{\mathrm{g}}\right)$ of the bulk material using a Mettler Toledo DSC $822 \mathrm{e}$ with a temperature ramp from 50 to $200{ }^{\circ} \mathrm{C}$ at a rate of $10{ }^{\circ} \mathrm{C} / \mathrm{min}$ over two runs. The glassy- and rubbery-state regions before and after the transition were linearly fitted with a third fit imposed tangentially to the point of inflection in the transition. Intersections of the tangent with the glassy- and rubbery-state regions correspond to the onset and endpoint $T_{g}$ values, respectively, while the inflection point between these two values is presented as the $T_{g}$ of the material.

Fluorescence experiments were performed with an Edinburgh Instruments Co. fluorimeter. Temperature was controlled with a Perkin Elmer DSC SC7 and TAC 7/DX Thermal Analysis Controller. Samples were first heated to $200{ }^{\circ} \mathrm{C}$ for $10 \mathrm{~min}$ to erase their thermal history and then cooled to $50{ }^{\circ} \mathrm{C}$ at a $100{ }^{\circ} \mathrm{C} / \mathrm{min}$ cooling rate. An optic fiber was used to both excite and collect the fluorescence of the sample between 345 and $650 \mathrm{~nm}$ with an excitation wavelength of $340 \mathrm{~nm}$. Excitation and emission slits were set between 1.75 and $2.25 \mathrm{~nm}$ to insure an adequate response, but not 
exceeding a maximum intensity of $10^{4}$ counts. Fluorescence experiments were performed in a stepwise manner: temperatures between 50 and $90{ }^{\circ} \mathrm{C}$, as well as between 130 and $160{ }^{\circ} \mathrm{C}$, were increased by $5{ }^{\circ} \mathrm{C}$ increments $\left(10^{\circ} \mathrm{C} / \mathrm{min}\right.$ heating rate) and allowed to equilibrate for $2 \mathrm{~min}$; between 90 and $130{ }^{\circ} \mathrm{C}$ the increments were decreased to $3{ }^{\circ} \mathrm{C}$. Fluorescence spectra were taken after each equilibration time.

\subsubsection{Scanning electron microscopy}

Fracture surfaces were inspected with a Phillips XL30 scanning electron microscope to observe the microstructure in the nanocomposites. Samples were prepared by submerging them in liquid nitrogen, breaking it, and sputter-coating it with gold. Accelerating voltages between 10 and $13 \mathrm{kV}$ were used to capture secondary and backscattered electron images.

\section{Results and discussion}

\subsection{Silica nanoparticle characterization}

After coating and drying the silica nanoparticles, FTIR was used to determine if the silane had covalently attached to the silica nanoparticle surface while TGA was used to quantitatively determine the quantity attached. Fig. 2 shows the FTIR spectra of treated and untreated nanoparticles on the same plot. Observation of the characteristic peaks at 3430 and $1623 \mathrm{~cm}^{-1}(-\mathrm{O}-\mathrm{H}$ stretching) and at $801 \mathrm{~cm}^{-1}$ ( $\mathrm{Si}-\mathrm{O}-\mathrm{H}$ bending) confirms the presence of silica, and the bands at $1250 \mathrm{~cm}^{-1}$ for $\mathrm{Si}-\mathrm{CH}_{2}$ stretching and at $2960 \mathrm{~cm}^{-1}$ for $\mathrm{C}-\mathrm{H}$ stretching indicate the presence of the silane agents. An additional peak at $2877 \mathrm{~cm}^{-1}$ for $-\mathrm{C}-\mathrm{H}$ stretching is present in nanoparticles treated with GPMES. While these spectra indicate that there is silane present in the dried nanoparticle samples, the silica signal is saturated indicating that there is far less silane present compared to the quantity of silica.

A quantitative analysis of the silane coverage on the nanoparticle surfaces was performed by analyzing the TGA curves, which are shown in Fig. 3. Using the untreated silica curve as the reference and taking the remaining weight difference between 200 and $800{ }^{\circ} \mathrm{C}(\Delta w)$, the following calculation was performed: [28]

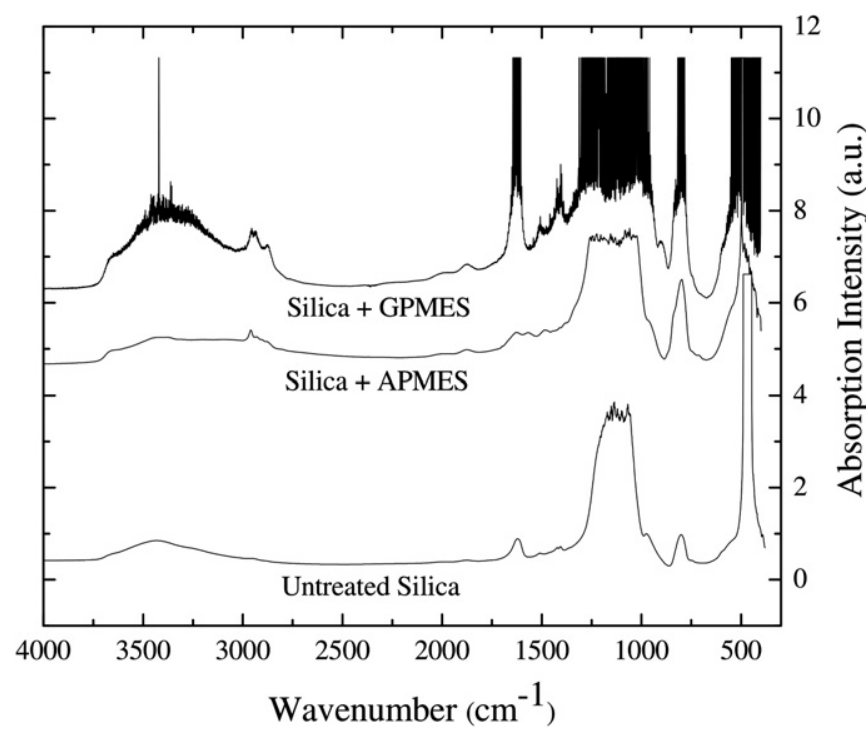

Fig. 2. FTIR spectra of bare silica, silica treated with APMES, and silica treated with GPMES.

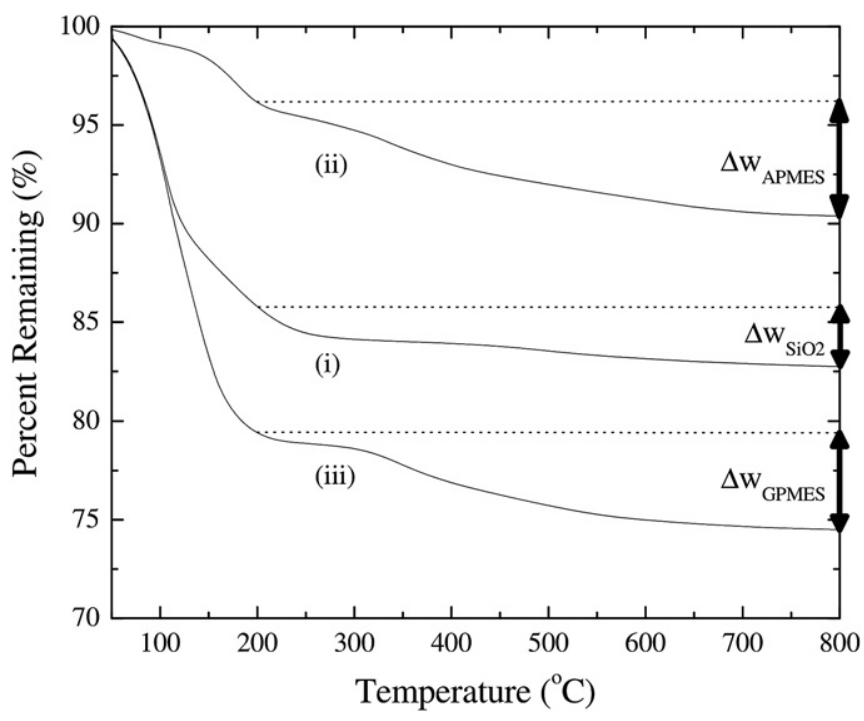

Fig. 3. TGA curves of samples in Fig. 2: (i) bare silica, (ii) silica treated with APMES, (iii) silica treated with GPMES. The data were analyzed above $200{ }^{\circ} \mathrm{C}$ to insure that all remaining solvent in the system had evaporated.

$\frac{\Delta w_{\mathrm{ModSiO}_{2}}-\Delta w_{\mathrm{SiO}_{2}}}{100 \cdot M_{\mathrm{Mod}} \cdot S}$

where $M_{\text {Mod }}$ is the molecular mass that is lost as volatile gas between 200 and $800{ }^{\circ} \mathrm{C}$ per mol of used silane $(144.21 \mathrm{~g} / \mathrm{mol}$ for GPMES and $88.17 \mathrm{~g} / \mathrm{mol}$ for APMES), and $s$ is the specific surface area of the silica nanoparticles $\left(190 \mathrm{~m}^{2} / \mathrm{g}\right)$. This calculation yields values of $1.47 \mu \mathrm{mol} / \mathrm{m}^{2}$ for APMES and $0.96 \mu \mathrm{mol} / \mathrm{m}^{2}$ for GPMES. Assuming 4.6 silanol groups[41] per $\mathrm{nm}^{2}$, the monolayer coverage extent becomes $13 \%$ for GPMES and $19 \%$ for APMES. Low monolayer coverage indicates that fluorophores will not be in close contact with each other, and thus interactions between fluorophores can be neglected.

\section{2. $\mathrm{PMMA} / \mathrm{SiO}_{2}$ nanocomposite characterization}

DMAc has a boiling point of $160{ }^{\circ} \mathrm{C}$, and therefore, requires high temperatures and long durations under vacuum to be removed through casting, resulting in the possible formation of aggregates and quenching of the fluorophore signal. During precipitation, the solvent is rejected from the nanocomposite due to the concentration gradient produced by the presence of the methanol phase. In theory, the absence of solvent allows the polymer to fall out of solution and encapsulate the silica nanoparticles. However, because there is a concentration gradient across the phases, other components in the system potentially may diffuse into the methanol phase. To determine the species ejected from the nanocomposite solution, the filtered methanol was centrifuged and the supernatant discarded. The remaining pellet was vacuum dried and characterized with FTIR. As seen in Fig. 4, and from the data of Wang et al. [42], the signature from the pellet possesses many peaks reflective of the presence of PMMA in the pellet, but due to overlaps in the signals it can only be inferred that silica is not the primary component in the pellet.

Although the stoichiometry was set to produce 2 and 5\% silica in the final nanocomposites, because of solution processing, it is possible that deviation from these initial values took place. The determination of the actual amount of silica in the nanocomposite was obtained via TGA experiments. The amount of silica deviated between different composites but average percentages by weight 


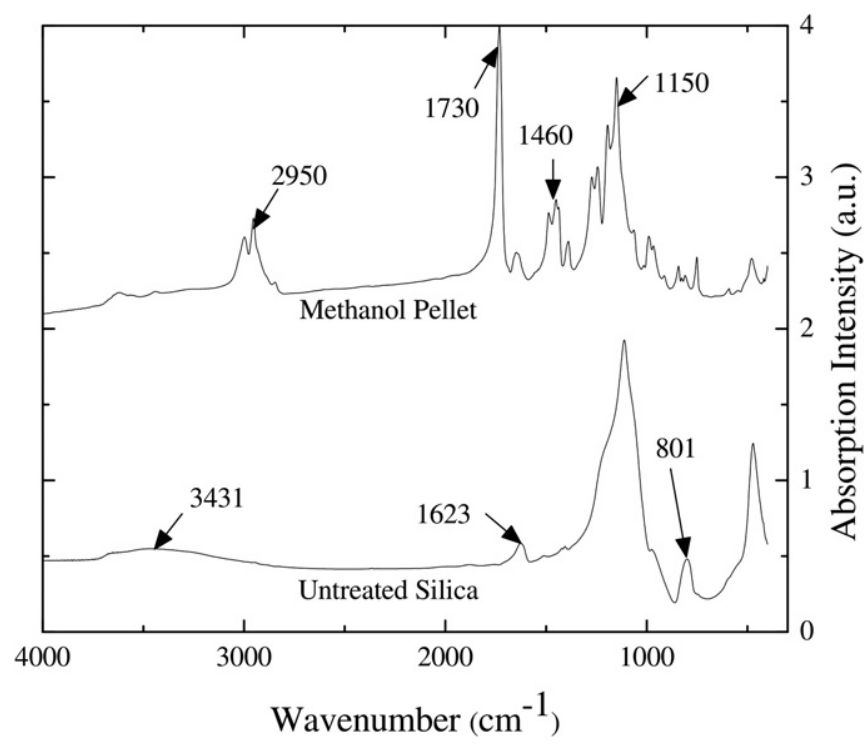

Fig. 4. FTIR spectra of bare silica nanoparticles and solids suspended in the methanol bath fluid.

were as follows: $1.12 \%( \pm 0.57)$ instead of $2 \%$, and $5.29 \%( \pm 0.75)$ instead of $5 \%$. These results indicate that the precipitation method did effectively produce PMMA/silica nanocomposites but with small deviations in the final concentrations.

\subsection{Nanocomposite thermal analysis}

\subsubsection{Differential scanning calorimetry}

The results of the classical bulk DSC analysis showed that the $T_{g}$ of the nanocomposite is higher compared to the neat PMMA (Table 1). Neat PMMA has a bulk $\mathrm{T}_{\mathrm{g}}$ of $114.6{ }^{\circ} \mathrm{C}$ while the nanocomposite has a $\mathrm{T}_{\mathrm{g}}$ value of $123.9{ }^{\circ} \mathrm{C}$ for the $2 \%$ silica containing composite (the average of all $2 \%$ samples $\left(2 \%-\mathrm{SiO}_{2} / \mathrm{PMMA}\right.$, N2ADNS-Cl, N2GJ230DNS-Cl, N2GJ400DNS-Cl) is $124.1 \pm 1.2{ }^{\circ} \mathrm{C}$ ) and $122.1{ }^{\circ} \mathrm{C}$ for $5 \%$ silica containing sample (with an average value of $124.3 \pm 1.6{ }^{\circ} \mathrm{C}$ for all $5 \%$ samples (5\%-SiO $2 / P M M A, N 5 A D N S-C l$, N5GJ230DNS- $C l, N 5 G J 400 D N S-C l)$ ). Given that the standard error for a DSC experiment is around $1{ }^{\circ} \mathrm{C}$, the $\mathrm{T}_{\mathrm{g}}$ values obtained for different samples are quite close to each other. In addition, it was previously stated that not all samples contain the same amount of silica, which might be the reason for these small deviations. It is important, however, that the addition of a surface modifier does not have any effect on the interaction of PMMA with the silica surface. The PMMA ester side groups form hydrogen bonds with the silanol groups of the silica. It is not expected that the surface modification in this work influences PMMA-silica interactions because of the very small amount of surface modifiers used.

Table 1

Summary of $T_{g}$ values obtained from the second thermal scan during DSC experiments. The $\mathrm{T}_{\mathrm{g}}$ of the neat PMMA is $114.6{ }^{\circ} \mathrm{C}\left(\mathrm{T}_{\mathrm{g} \text { onset }}=104.7{ }^{\circ} \mathrm{C}, \mathrm{T}_{\mathrm{g} \text { end }}=124.5^{\circ} \mathrm{C}\right.$, $\Delta \mathrm{T}=19.8^{\circ} \mathrm{C}$ ).

\begin{tabular}{|c|c|c|c|c|c|c|c|c|}
\hline \multirow[t]{3}{*}{ Sample } & \multicolumn{8}{|l|}{$\mathrm{T}_{\mathrm{g}}\left({ }^{\circ} \mathrm{C}\right)$} \\
\hline & \multicolumn{4}{|c|}{$2 \% \mathrm{SiO}_{2}$} & \multicolumn{4}{|l|}{$5 \% \mathrm{SiO}_{2}$} \\
\hline & $\mathrm{T}_{\text {g,onset }}$ & $\mathrm{T}_{\mathrm{g}, \mathrm{mid}}$ & $T_{g \text {,end }}$ & $\Delta \mathrm{T}$ & $\mathrm{T}_{\text {g,onset }}$ & $\mathrm{T}_{\mathrm{g}, \mathrm{mid}}$ & $T_{\text {g,end }}$ & $\Delta \mathrm{T}$ \\
\hline $\mathrm{PMMA} / \mathrm{SiO}_{2}$ & 118.2 & 123.9 & 129.5 & 11.4 & 115.7 & 122.1 & 128.4 & 12.7 \\
\hline$N \times A D N S-C l$ & 117.1 & 123.2 & 129.2 & 12.1 & 120.4 & 125.4 & 130.4 & 10.1 \\
\hline NxGJ230DNS-Cl & 121.3 & 126.2 & 131.1 & 9.8 & 117.4 & 123.3 & 129.2 & 11.8 \\
\hline $\mathrm{NxGJ400DNS-Cl}$ & 118.2 & 124.0 & 129.7 & 11.5 & 119.4 & 124.9 & 130.3 & 10.9 \\
\hline
\end{tabular}

Standard error in all DSC measurements is $\pm 1^{\circ} \mathrm{C}$
For the case of nanosilica samples functionalized with polypropylene glycol, miscibility of the tether (polypropylene glycol) with the PMMA matrix may determine the wetting of the surface by the PMMA. But PMMA and polypropylene glycol are known to be miscible at temperatures below the $T_{g}$ of the PMMA, especially for low molecular weight polypropylene glycol [43]. Therefore, the modifiers should not prevent the PMMA chains to access the nanoparticle surface.

The $T_{g}$ increase observed upon addition of silica to PMMA does not depend on the amount of silica present in the system. This is because the silica concentrations used in the current study are higher than the critical concentration around which a large impact on $\mathrm{T}_{\mathrm{g}}$ is observed, as indicated by Ash et al. [28]

\subsubsection{Fluorescence spectroscopy}

The fluorescence emission spectra indicate that there is a significant red shift and decrease in signal intensity as the temperature increases, indicating that both the first moment of emission band, $\langle\nu\rangle$, and the integrated intensities may be used to determine the transitions in the material (Fig. 5). For direct comparison between samples, the integrated intensity for each sample was normalized to the intensity at $50{ }^{\circ} \mathrm{C}$. Fig. 6 illustrates the typical temperature dependence of both photophysical variables and the characteristic change near the $\mathrm{T}_{\mathrm{g}}$ value. The glassyand rubbery-state regions at temperatures well before the $T_{g}$ were linearly fitted, with the intersection of the fits representing the $T_{g}$ of the system (Fig. 6), following the method employed by Torkelson [18]. Onset and endpoint $T_{g}$ values are identified as the points at which the data diverge from the linear fits.

The steady-state $T_{g}$ values as a function of maximum surface modifier length for the two concentrations used as well as the $\mathrm{T}_{\mathrm{g}}$ values of the matrix as detected by the two references used in this work ( $R_{\text {Short }}$ and $R_{\text {Long }}$ ) are shown in Fig. 7 . The $T_{g}$ values of bulk PMMA detected by the two references using the fluorescent intensity criteria are $113^{\circ} \mathrm{C}\left(R_{\text {Short }}\right)$ and $107^{\circ} \mathrm{C}\left(R_{\text {Long }}\right)$; using the first moment criteria the values are $111{ }^{\circ} \mathrm{C}\left(R_{\text {Short }}\right)$ and $108{ }^{\circ} \mathrm{C}\left(R_{\text {Long }}\right)$. These values are all lower than the $\mathrm{T}_{\mathrm{g}}$ measured by DSC. This fact can be attributed to the different heating rates used in the two experiments; about $1.3 \mathrm{~K} / \mathrm{min}$ for fluorescence (within 90 and $130{ }^{\circ} \mathrm{C}$ region, where a $3{ }^{\circ} \mathrm{C}$ heating increments and 2 min equilibration time was used) and $10 \mathrm{~K} / \mathrm{min}$ for DSC and according to data from Karlou [44] who measured $\mathrm{T}_{\mathrm{g}}$ of a similar PMMA used in the

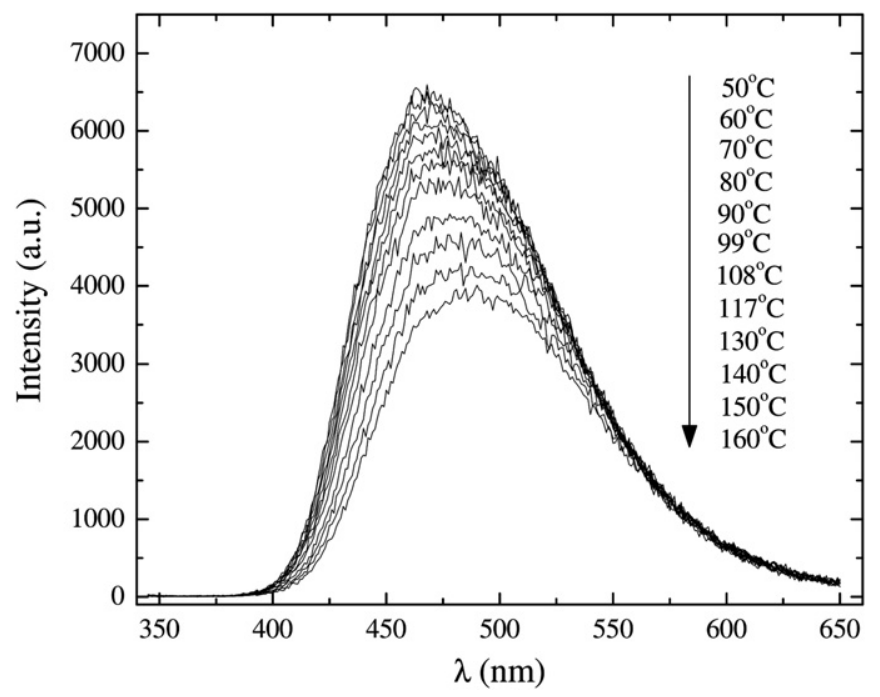

Fig. 5. Emission spectra of steady-state measurements of neat PMMA doped with DNS-Cl bound to APMES (PADNS-Cl). 


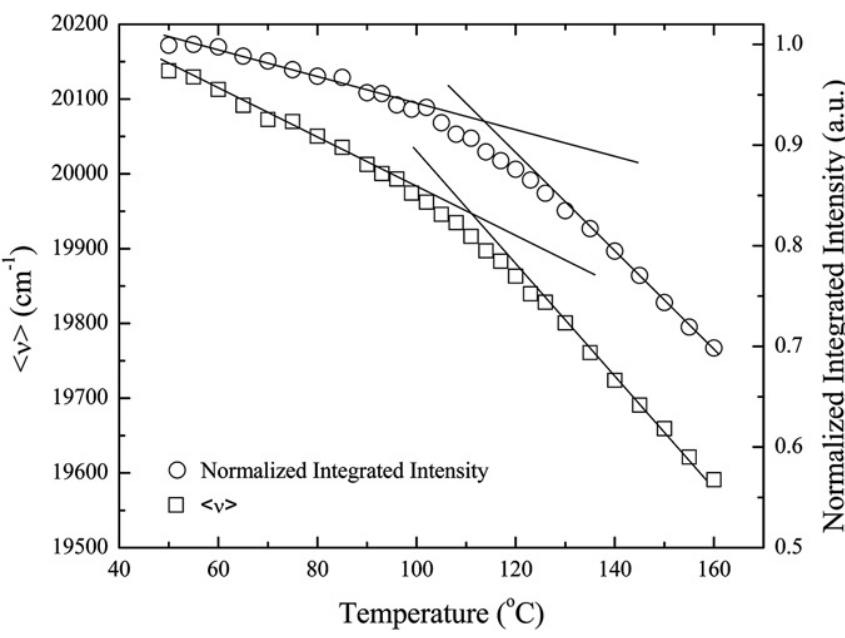

Fig. 6. Temperature dependence of the first moment, $\langle\nu\rangle$, (open squares) and the integrated intensity (open circles) of fluorescence spectra of the neat PMMA doped with APMES bound to DNS-Cl (PADNS-Cl).

current work at different heating rates. Nevertheless, fluorescence measurements do not necessarily match DSC measurements since the two techniques differ in the basic physical mechanism and in the corresponding characteristic length scale.

The most important aspect of Fig. 7 is that the observed $T_{g}$ decreases as the length of the molecular spacer is increased. Decreasing $\mathrm{T}_{\mathrm{g}}$ values as a function of spacer length were anticipated as the hydrogen bonds between the hydroxyl group on the silica and oxygen from the ester group on the PMMA reduce both the mobility and the free volume near the polymer-nanoparticle interface, resulting in a greater $\mathrm{T}_{\mathrm{g}}$ at the interface compared to that of the bulk polymer. As the distance between the silica surface and the fluorophore increases, both the free volume and the mobility in the local environment of the fluorophore increases leading to a decrease in the $T_{g}$ towards that of the neat polymer. Examination of the $\langle\nu\rangle$ vs. graft length shows no significant difference in $T_{g}$ values between the two silica concentrations ( 2 and $5 \%$ ) with the exception of the shortest surface modifier. However, results from the integrated intensity indicate that the $2 \%$ silica samples exhibit significantly greater $\mathrm{T}_{\mathrm{g}}$ values than the $5 \%$ samples with increasing graft length for samples with DNS-Cl fluorophores. This finding might be due to agglomeration of the silica nanoparticles at the higher concentration. In fact, SEM images indicate that there is some agglomeration in the $5 \%$ silica containing samples (Fig. 8 ).

In these experiments, the position of the fluorophore is not well defined with respect to the nanoparticle surface. Due to the presence of oxygen in the backbone of the molecular spacer, the spacer chains have high flexibility and thus the position of the fluorophores varies; therefore, in actuality a distribution of fluorophore-silica distances exists. This hinders the accuracy of correlating the length of the graft with the $T_{g}$ value as the length of the graft does not necessarily directly correlate with the distance of the fluorophore from the nanoparticle surface. With a larger chain length, the number of chain configurations increases, which increases the distribution of relaxed and non-relaxed states within a given sample, causing an increase in the width of the $T_{g}$ range. However, this trend was not observed in the current study.

Upon examination of the variation in the first moment within the temperature range studied in the current work, it is observed that in all cases this value is about $500 \mathrm{~cm}^{-1}$, which is much higher than the thermal energy difference between the initial and final temperatures $\left(70 \mathrm{~cm}^{-1}\right)$, indicating that the excited dansyl moieties
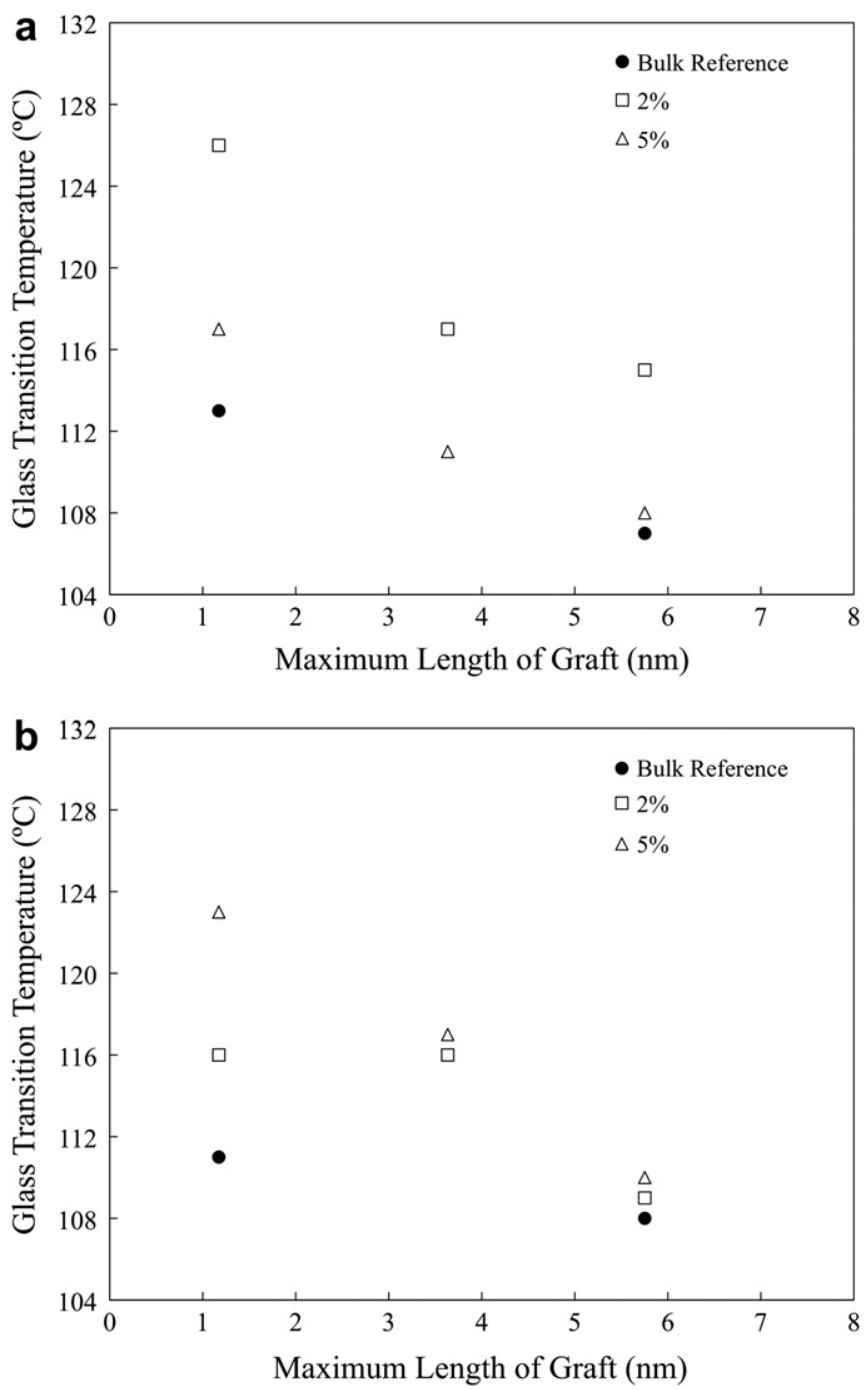

Fig. 7. $T_{g}$ values from steady-state measurements from the (a) integrated intensity and (b) first moment of the emission spectra, $\langle\nu\rangle$. Bulk reference values represent the $T_{g}$ obtained from systems where the fluorophore attached to the silane coupling agent and (if necessary) Jeffamine was dispersed in neat PMMA. Steady-state measurements were performed by increasing the temperature from 50 to $160{ }^{\circ} \mathrm{C}$ by $5^{\circ} \mathrm{C}$ steps $\left(3{ }^{\circ} \mathrm{C}\right.$ between 90 and $130{ }^{\circ} \mathrm{C}$ ) and allowing the sample to equilibrate for $2 \mathrm{~min}$ after each step.

are in a more relaxed state after the glass transition of the PMMA matrix. The relaxation of the excited state of the fluorophore is a consequence of a complex combination of dipolar and vibronic couplings with the environment. Nevertheless, attempts to correlate the extent of relaxation with the spacer length failed. For example, at low temperatures $\left(50^{\circ} \mathrm{C}\right)$ the average value of $\langle\nu\rangle$ for the samples containing the fluorophore attached to the silica surface is $20298 \pm 34 \mathrm{~cm}^{-1}$ while the corresponding value for the reference $R_{\text {Short }}$ dissolved in the bulk is $20137 \mathrm{~cm}^{-1}$; the difference between these two values, $161 \mathrm{~cm}^{-1}$, although small, is significant and might indicate that fluorophores attached to the surface of silica particles are in a more rigid environment that hinders their excited state relaxation to the same extent as in the bulk. But the value obtained for the reference $R_{\text {Long }}$ is $2354 \mathrm{~cm}^{-1}$, in the same range as grafted chromophores. At high temperature $\left(150^{\circ} \mathrm{C}\right)$, those fluorescent moieties located very near the surface, separated only by a silane coupling molecule, have a high value, around $19772 \pm 88 \mathrm{~cm}^{-1}$; those moieties with a large spacer have lower 

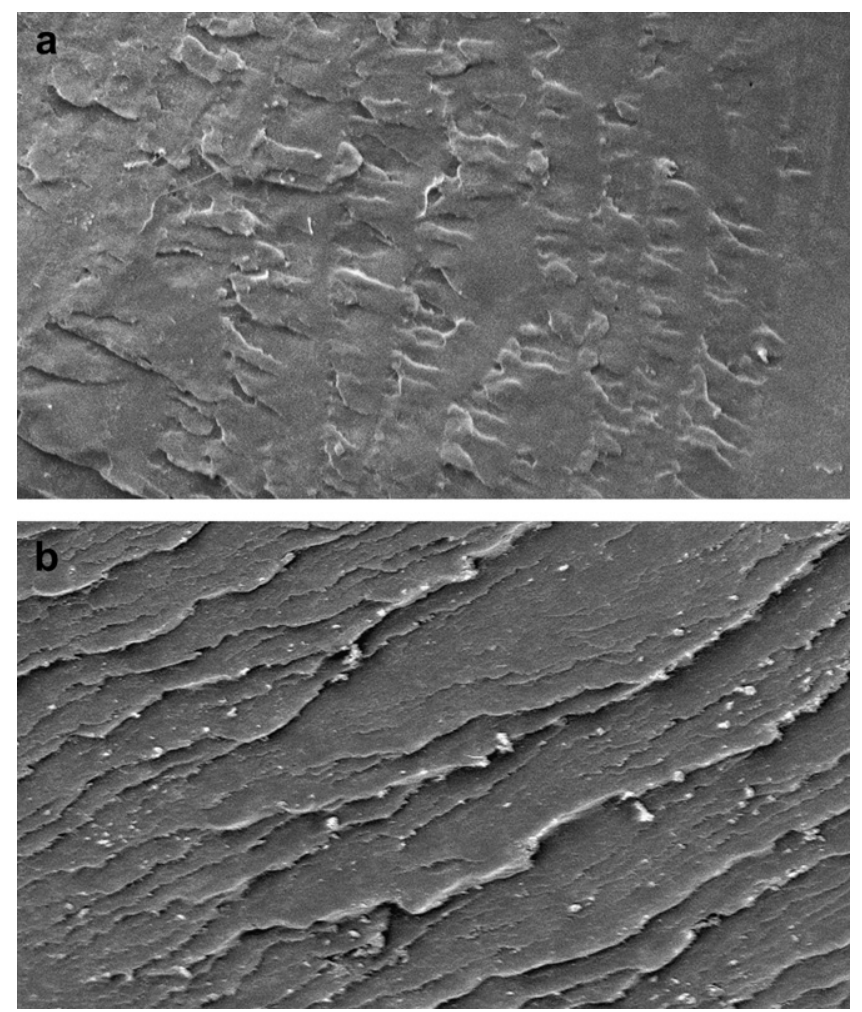

Fig. 8. SEM micrographs of PMMA with (a) $2 \%$ and (b) $5 \%$ silica nanoparticles treated with GPMES, Jeffamine D-230, and DNS-Cl (NxGJ230DNS-Cl). Width of the figure corresponds to $250 \mu \mathrm{m}$.

$<\nu>$ values, about $19660 \pm 12 \mathrm{~cm}^{-1}$. But, again, the average energy of the emission for the two references ( $R_{\text {Short }}$ and $R_{\text {Long }}$ ) presents an opposite trend: $R_{\text {Short }}$ has the lowest value, around $19591 \mathrm{~cm}^{-1}$ and $R_{\text {Long }}$ has a high value of $19827 \mathrm{~cm}^{-1}$. The lack of correlation suggests that the local environment of each chromophore is different and strongly dependent on the nature of the chains directly attached to it.

Therefore, comparison between $\mathrm{T}_{\mathrm{g}}$ values obtained from fluorophores bearing different spacers must be done with caution. A more proper comparison can be made between samples bearing the same spacer both dissolved in the bulk or grafted to the silica surface. Hence, $\mathrm{NxADNS}-\mathrm{Cl}$ must be compared with reference $R_{\text {Short }}$

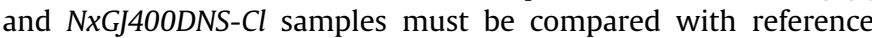
$R_{\text {Long. }}$ Reanalysis of Fig. 7 shows that the $\mathrm{T}_{\mathrm{g}}$ of grafted samples is higher than its corresponding reference. Moreover, the longer the spacer, the smaller the difference with the corresponding reference. This result confirms that as the fluorophore is in closer proximity to the surface of the silica particles, the environment is more rigid and presents a higher $\mathrm{T}_{\mathrm{g}}$. The comparison of the experimental values with reference values also suggests that around $\sim 7 \mathrm{~nm}$ (calculated from $2 \%$ results), the $T_{g}$ observed by the fluorophore is the same as the bulk $\mathrm{T}_{\mathrm{g}}$; hence, this distance can be assumed to be the interfacial thickness (as defined with respect to $T_{g}$ ). This distance agrees well with the immobilized layer thickness that was calculated by Chen et al., [29] although the two definitions do not necessarily agree with each other. In the current work, the interfacial distance is defined directly from $T_{g}$ change, whereas Chen et al. defined the immobilized region thickness from a structural point of view (approximate thickness of the amorphous layer around the nanoparticle).

One final issue is the effect of the presence of the Jeffamine molecular spacers (polypropylene glycol) in the vicinity of the fluorescence probe. Because the probe is sensitive to its environment and
Jeffamine is part of this environment, then it is necessary to question if the $T_{g}$ measured by the probe is affected by the $T_{g}$ of the Jeffamine. The $\mathrm{T}_{\mathrm{g}} \mathrm{S}$ of Jeffamines at various molecular weights were reported by Leon et al. [45] to be $-105,-78,-80$, and $-75^{\circ} \mathrm{C}$ for $1,2,3$, and 69 repeat units, respectively. On the other hand, the $\mathrm{T}_{\mathrm{g}}$ of the PMMA used in this study is approximately $115{ }^{\circ} \mathrm{C}$. Therefore, if the presence of the Jeffamine affected the results, then the $\mathrm{T}_{\mathrm{g}}$ that is observed by the fluorescent probe should be lower compared to the real $T_{g}$ of the PMMA.

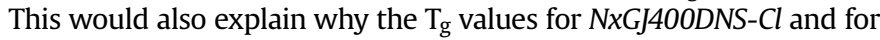
reference $R_{\text {Long }}$ reported in Fig. 7 are slightly lower than the bulk $\mathrm{T}_{\mathrm{g}}$ value detected by the reference $R_{\text {Short }}$. On the other hand, this finding also suggests that the presence of Jeffamine did not result in the observed increase of $\mathrm{T}_{\mathrm{g}}$ as the probe approached the silica surface, but rather, the observed $\mathrm{T}_{\mathrm{g}}$ is due to the interfacial effects as proposed in the current study.

\section{Conclusions}

The glass transition temperatures of silica/PMMA nanocomposites were measured in the bulk through differential scanning calorimetry and as a function of distance from the silica-PMMA interface through fluorescence spectroscopy. As far as the authors know, this is the first study that probes the local $\mathrm{T}_{\mathrm{g}}$ at the interface of a polymer and a nanoparticle in a polymer nanocomposite directly, and in the present case showing a gradual decrease in the glass transition temperature as a function of distance from the nanoparticle-polymer interface. The glass transition temperature of the nanocomposites was found to be on average $10^{\circ} \mathrm{C}$ greater than that of the neat polymer. No dependence on nanosilica concentration was identified in the concentration range studied.

Fluorescence spectroscopy indicated that the glass transition temperature of the nanocomposite decreased approaching the bulk value of the polymer as the size of the molecular spacer increased, illustrating that there is a glass transition temperature gradient within the local environment surrounding the nanofiller. Both the integrated intensity and the first moment of the fluorescence spectra, $\langle\nu\rangle$, demonstrated this decrease in $\mathrm{T}_{\mathrm{g}}$.

Flexibility of the molecular spacers increases the distribution of fluorophore distance from the interface, preventing a direct correlation between the spacer length and distance from the nanoparticle surface. If it is assumed that the molecular spacers are completely stretched out into the bulk, then the results indicate that the glass transition temperature reaches the bulk value in less than $\sim 7 \mathrm{~nm}$. Even if this assumption (fully stretched spacers) is not accurate, $7 \mathrm{~nm}$ presents a maximum distance that the effect of the interface extends into the bulk.

The distribution of fluorophore distance from the nanoparticle surface also allows for polarity effects to potentially obscure the response of $\langle\nu\rangle$ to changes in rigidity. Fluorophores similar in microstructure exhibit different responses to the local environment and cannot be used to directly correlate glass transition temperatures as a function of the distance from the nanoparticle surface.

\section{Acknowledgements}

This research was supported by the National Science Foundation under Grant No. 0500324. Authors from UC3M would like to acknowledge CICYT for financial support (MAT 2007-63722).

\section{References}

[1] Adam G, Gibbs JH. J Chem Phys 1965;43:139.

[2] Jackson CL, McKenna GB. J Non-Cryst Solids 1991;131-133:221.

[3] Kalogeras IM, Neagu ER. Eur Phys J E 2004;14:193.

[4] Schönhals A, Goering H, Schick Ch, Frick B, Zorn R. Eur Phys J E 2003;12:173. 
[5] Schönhals A, Goering H, Schick Ch, Frick B, Zorn R. J Non-Cryst Solids 2005;351:2668.

[6] Alcoutlabi M, McKenna GB. J Phys Condens Matter 2005;17:R461.

[7] Keddie JL, Jones RAL, Cory RA. Europhys Lett 1994;27:59.

[8] Mattsson J, Forrest J, Borjesson L. Phys Rev E 2000;62:5187.

[9] Mundra MK, Donthu SK, Dravid VP, Torkelson JM. Nano Lett 2007;7:713.

[10] Mundra MK, Ellison CJ, Rittigstein P, Torkelson JM. Eur Phys J Spec Top 2007; $141: 143$.

[11] Roth CB, Dutcher JR. Eur Phys J E 2003;12:S102.

[12] Dalnoki-Veress K, Forrest JA, Murray C, Gigault C, Dutcher JR. Phys Rev E 2001;63:031801-1.

[13] Singh L, Lodovice PJ, Henderson CL. Thin Solid Films 2004;449:231.

[14] Sills S, Overney RM, Chau W, Lee VY, Miller RD, Frommer J. Chem Phys 2004; 120:5334.

[15] Forrest JA, Mattsson J. Phys Rev E 2000;61:R53.

[16] Roth CB, McNerny KL, Jager WF, Torkelson JM. Macromolecules 2007;40:2568.

[17] Ellison CJ, Torkelson JM. Nat Mater 2003;2:695.

[18] Ellison CJ, Torkelson JM. J Polym Sci Part B Polym Phys 2002;40:2745.

[19] Rittigstein P, Priestley RD, Broadbelt LJ, Torkelson JM. Nat Mater 2007;6:278.

[20] Herminghaus S, Jacobs K, Seeman R. Eur Phys J E 2001;5:531.

[21] Rittigstein P, Torkelson JM. J Polym Sci Part B Polym Phys 2006;44:935.

[22] Long D, Lequeux F. Eur Phys J E 2001;4:371.

[23] Long D. Eur Phys J E 2002;8:245.

[24] Ngai KL, Rizos AK, Plazek DJ. J Non-Cryst Solids 1998;235-237:435

[25] Ngai KL. Eur Phys J E 2002;8:225.

[26] de Gennes PG. Eur Phys J E 2000;2:201

[27] Krishnamoorti R, Vaia RA, Giannelis EP. Chem Mater 1996;8:1728.

[28] Ash BJ, Siegel RW, Schadler LS. J Polym Sci Part B Polym Phys 2004;42:4371.

[29] Chen L, Zheng K, Tian X, Hu K, Wang R, Liu C, et al. Macromolecules 2010;43:1076.

[30] Srivastava S, Basu JK. Phys Rev Lett 2007;98:165701.

[31] Bansal A, Yang H, Li C, Cho K, Benicewicz BC. Nat Mater 2005;4:693.

[32] Lee J-Y, Su KE, Chan EP, Zhang Q, Emrick T, Crosby AJ. Macromolecules 2007; $40: 7755$.

[33] Roth CB, Torkelson JM. Macromolecules 2007:40:3328.

[34] Gonzalez-Benito J, Olmos D, Sanchez PG, Aznar AJ, Baselga J. J Mat Proc Tech 2003;143-144:153.

[35] Peinado C, Salvador EF, Baselga J, Catalina F. Macromol Chem Phys 2001;202:1924.

[36] Peinado C, Alonso A, Salvador EF, Baselga J, Fernando C. Polymer 2002;43:5355.

[37] Mikes F, Gonzalez-Benito J, Baselga J. J Macromol Sci Phys 2001;B40:405.

[38] Albala R, Olmos D, Aznar AJ, Baselga J, Gonzalez-Benito J. J Coll Int Sci 2004;277:71.

[39] Turrion SG, Olmos D, Ekizoglu N, Gonzalez-Benito J. Polymer 2005;46:4023.

[40] Bergna HE, Roberts WO, editors. Colloidal silica: fundamentals and applications. Boca Raton: CRC; 2006. p. 28.

[41] De Boer JH, Vleeskens JM. Proc K Ned Akad Wet Ser B 1958;61(2). Zhuravlev, L.T., Colloids Surf 1993, 74, 71.

[42] Wang L, Chen D. Colloid Polym Sci 2006;284:449.

[43] Jaffrennou B, Soule ER, Mechin F, Borrajo J, Pascault JP, Williams RJJ. Polymer 2000;45:7185.

[44] Karlou K, Schneider HA. J Therm Anal Cal 2000;59:59.

[45] León C, Ngai KL, Roland CM. J Chem Phys 1999;110:11585. 